\title{
Fracture risk assessment of female thyroid patients in Damietta
}

\author{
Noreya M. Abeed ${ }^{1,3}$, Ashraf Abdul-Salam ${ }^{2}$, Hekmat L. El-Gammal ${ }^{1}$ and Ayman Hyder ${ }^{1}$ \\ 1- Faculty of Science, Damietta University, Egypt. \\ 2- Faculty of Medicine, Azhar University, New Damietta, Egypt \\ 3- Faculty of Medical Technology, Tripoli University, Libya
}

\begin{abstract}
The clinical relevance of osteoporosis depends on the increase of fracture rate. The fracture risk assessment tool (FRAX) is a widely used tool to calculate the risk of fracture. The FRAX tool integrates bone mineral density (BMD) at the femoral neck only to calculate the 10-year fracture risk estimate, neglecting spine and forearm BMD. Focusing on the potential association between thyroid dysfunction and osteoporosis in Egyptian women, Damietta community, the purpose of the present study was to assess fracture risk by applying the FRAX tool in pre- and postmenopausal women with thyroid dysfunction, utilizing BMD data from spine and forearm. The results revealed that FRAX-score was gradually increased by the age in hypo- and hyperthyroid women, although the mean fracture risk was higher in hyperthyroid women in each of the lumbar spine and the forearm. All FRAX values were less than $10 \%$, indicating a low fracture risk in thyroid dysfunction. No significant differences have been observed when FRAX values calculated from BMD of different regions have been compared for the same age category. These results may indicate that BMD data from different regions can be recruited to calculate the corresponding FRAX values.
\end{abstract}

Key words: Fracture risk assessment, female thyroid patients, Damietta

\section{INTRODUCTION}

Osteoporosis is defined by the World Health Organization (WHO) as a skeletal disorder characterized by low bone mass combined with microarchitectural deterioration of the bone, leading to bone fragility and increased susceptibility to fracture. Its clinical relevance depends on the increasing of fracture rate, and on the morbidity due to loosing mobility and mortality associated with fractures, especially in the hip. The WHO defined osteoporosis based on bone mineral density (BMD). When the standard deviations of $\mathrm{BMD}$ is $\leq-2.5$ below peak bone mass (so-called T-score), as assessed by dual X-ray absorptiometry (DXA). A T-score between -1.0 and -2.5 means an intermediary condition of bone loss, which is called osteopenia. The risk of fracture increases significantly with decreasing BMD. However, it is well known that osteoporotic fractures occur across a wide spectrum of BMD intensity.
Actually, the much larger number of persons with osteopenia determines a significant occurrence of fractures in people diagnosed with this condition. There is no global consensus for screening patients at risk of osteoporotic fracture; but several medical associations recommend a targeted approach to the prevention of osteoporosis based on the 10-year absolute risk of osteoporotic fracture (Albergaria and Paula., 2019).

The main purpose of the treatments for osteoporosis is to decrease the risk of fragility fractures. Therefore, the capacity to assess the risk of fracture is critical for the identification and treating patients (Kanis, et al., 2005). The fracture risk assessment tool (FRAX), a computer based algorithm, is the most thoroughly studied and widely used tool to calculate the risk of fracture (Kanis et al., 2010). The FRAX was released in 2007 by the World Health Organization Collaborating Centre at Sheffield, United Kingdom, to 
Noreya M. Abeed et al.

estimate the individualized 10-year probability of hip and major osteoporotic fractures (hip, clinical spine, distal forearm, andshoulder(Kanis, 2007). The FRAX tool integrates 8 clinical risk factors: body mass index, previous fragility fracture, parental hip fracture, smoking, systemic glucocorticoid use, excess alcohol intake, rheumatoid arthritis, and other causes of secondary osteoporosis).These risk factors, in addition to age, sex and BMD at the femoral neck are used to calculate the 10year fracture risk estimate. However, the FRAX uses BMD at the femoral neck only, neglecting spine and shoulder BMD.

Numerous studies have focused on the potential association between thyroid dysfunction and osteoporosis or bone mineral density (BMD) Locally, there is no available data concerning the association between thyroid dysfunction and osteoporosis in pre and postmenopausal women in Egypt, especially in Damietta. Thus, the purpose of the present study was to assess fracture risk by applying the FRAX tool in pre- and postmenopausal women with thyroid dysfunction of Damietta community. In this study, the utilization of BMD data from spine and forearm in FRAX input was studied and compared that with the corresponding femur data.

\section{MATERIALS AND METHODS}

Egyptian women aged from 20 to 60 yearsold were enrolled in the preset study. The study included healthy control women and patients previously diagnosed with hyperthyroidism $(\mathrm{N}=25)$ or hypothyroidism $(\mathrm{N}=25)$. The study was conducted in Azhar University Educational Hospital, New Damietta, Egypt, which provides health services to the community of Damietta governorate. Participants were selected from patients with thyroid dysfunction who follow up their status in the hospital and volunteered to participate in the study. The previously diagnosis of hyperthyroidism and hypothyroidism was confirmed by biochemical estimations of serum TSH, FT3 and FT4.Other biochemical analyses of calcium, phosphorus and alkaline phosphatase were also performed.

\section{DXA-scanning:}

The principal use of dual-energy $\mathrm{X}$-ray absorptiometry (DXA) is to diagnose and monitor osteoporosis and therefore reduce fracture risk, associated morbidity, and mortality(Tanner and Moore,2012). The bone mineral density (BMD) scan was carried out by DXA scanner (Lunar Prodigy Primo, Encore, Version 17, Model 2016 USA for the tested women in Bone denistometry unit, Rheumatology Department, Azhar University Educational Hospital, New Damietta, Egypt. All women in the present study were investigated for osteoporotic fracture and BMD with DXA scanning of the lumbar spine, hip and wrist. DXA measures both bone mineral content (BMC, in grams) and area (in $\mathrm{cm} 2$ ). An "areal" BMD $(\mathrm{g} / \mathrm{cm} 2)$ is obtained by dividing bone mineral content by area. This value was converted to a $\mathrm{T}$ score or a $\mathrm{Z}$ score.

T-scores are calculated by taking the difference between a patient's measured BMD and the mean BMD in healthy young adults, matched for gender and ethnic group, and then dividing by the standard deviation (SD) of the reference population. When the study subjects are divided into quartiles on the basis of their baseline BMD measurements, an inverse relationship is found between fracture risk and BMD.

All tested women have been subjected to dual X-ray absorptiometry (DXA), to measure their bone densitometry of the lumbar spine, hip and wrist, a technique that is useful for measuring the bone mineral density (BMD) (Cummings et al., 2002). From their data, the risk of fracture was later estimated. The study protocol was conducted in accordance with the ethical 


\section{Fracture risk assessment of female thyroid patients in Damietta}

principles and guidance of the Helsinki Declaration. All participants agreed and provided a written consent to participate in the study.

\section{Anthropometric Characteristics:}

The participants were analyzed for their body mass index (BMI). detailed interviews have been performed with all participants and included questionnaire about demographic information such as age, educational qualification, type of family and occupation; information regarding clinical risk factors such as previous fracture at any site, vision capability, parents fracture, any diseases and medications; behavioral risk factors such as pattern of physical exercise, duration of exposure to sunlight, supplementation of calcium and vitamin D, cigarette smoking, alcohol consumption and caffeine intake in the form tea/coffee; and information related to the dietary intake like meal pattern, food consumption pattern and average nutrient intake. Some obtained data have been only used to assess the fracture risk.

\section{Fracture Risk Assessment (FRAX):}

In the present study, we applied the online calculation tool called Fracture Risk Assessment (FRAX). This tool uses selected clinical information as well as BMD to predict the 10-year probability of a hip fracture or a major osteoporotic fracture (spine, forearm, hip or shoulder fracture) in an individual. The tool was developed by the WHO Collaborating Centre for Metabolic Bone Diseases, University of Sheffield, UK, in collaboration with other scientific societies and is available at (https://www.sheffield.ac.uk/FRAX/tool.as px).As Egypt has not yet developed the own version of country specific calculation tool; the present study used the available calculation tool of Jordan as the nearest country for the FRAX risk score calculation.

\section{Statistical Analysis:}

Data are presented as mean \pm standard error of mean (SEM). Statistical differences between groups were evaluated using ANOVA followed by Student t-test. Differences between just 2 groups were evaluated by t-test. In all cases, the difference was considered statistically significant when the $\mathrm{p}$ value less than 0.05 .

\section{RESULTS}

Osteoporosis is diagnosed using DXA scan, which results in several sets of data including BMD, T-score and Z-score of several measured segments as L1-L4 of the spine, radius and ulna (forearm) and femur. The present technique of FRAX uses only $\mathrm{T}$-score from the femur to calculate the hip ten-year probability of fracture (\%) as well as the major (spine, hip and shoulder) osteoporotic fracture probability. However, it neglects the available data to calculate spine and forearm fracture probability using their available corresponding data from the DXA.The present work calculates FRAX from these available data of DXA measured in women with thyroid dysfunction. These women have been categorized into hypothyroid and hyperthyroid. Also, they were grouped according to their age.

The calculated FRAX values in hypothyroid women (Fig. 1) revealed an age-dependent increment. This was clear by tendency in spine and significantly only in femur. However, the results revealed low fracture probability in all cases under the effect of hypothyroidism, since the FRAX was not exceeding the $3 \%$ in any measured segment. Similarities in these FRAX ratios were obtained after calculations from the corresponding data of each organ. 
Noreya M. Abeed et al.

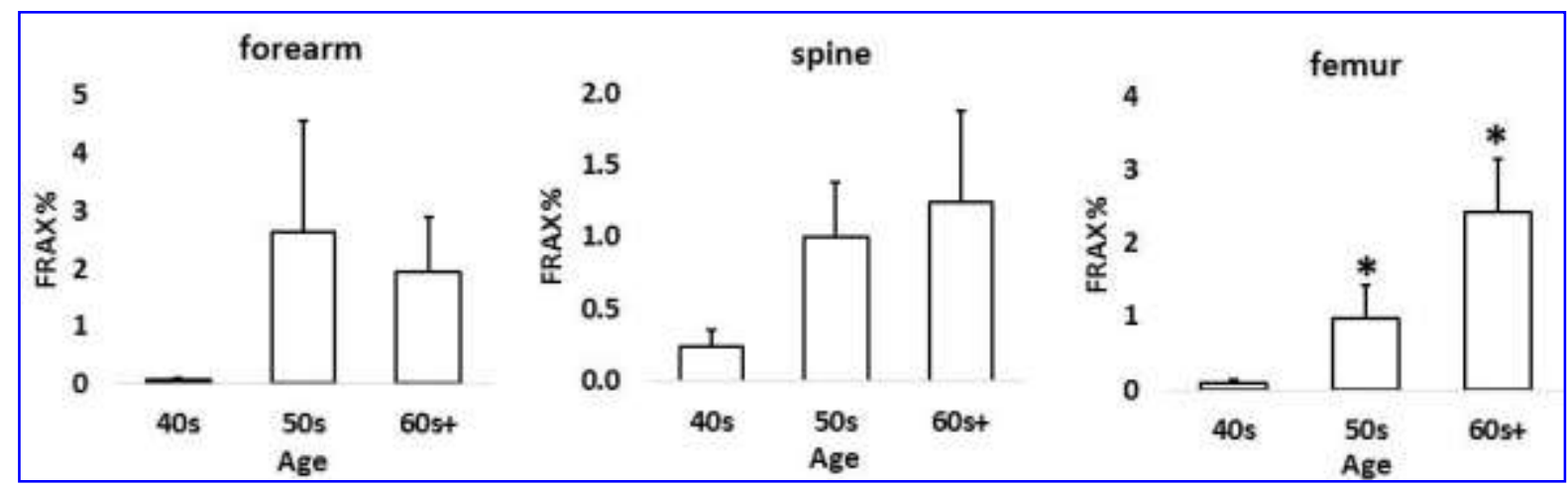

Fig. 1. Organ FRAX values as calculated from $T$-score of radius, spine and femur of hypothyroid women of different ages. Data are presented as mean $\pm \mathrm{SEM}$ of $\mathrm{N}=5$ from each age category. * denotes significantly different value from the value of the youngest.

Similarly, in case of hyperthyroidism, an age dependent increase in FRAX was obtained in the different measured segments after calculation from the specific correspondent data. Similar results were obtained for

spine and femur, with a gradual increase in FRAX as the age increases, while results of the forearm was a little bit different, with significant increase in FRAX in hyperthyroid women older than 60 years (Fig. 2).

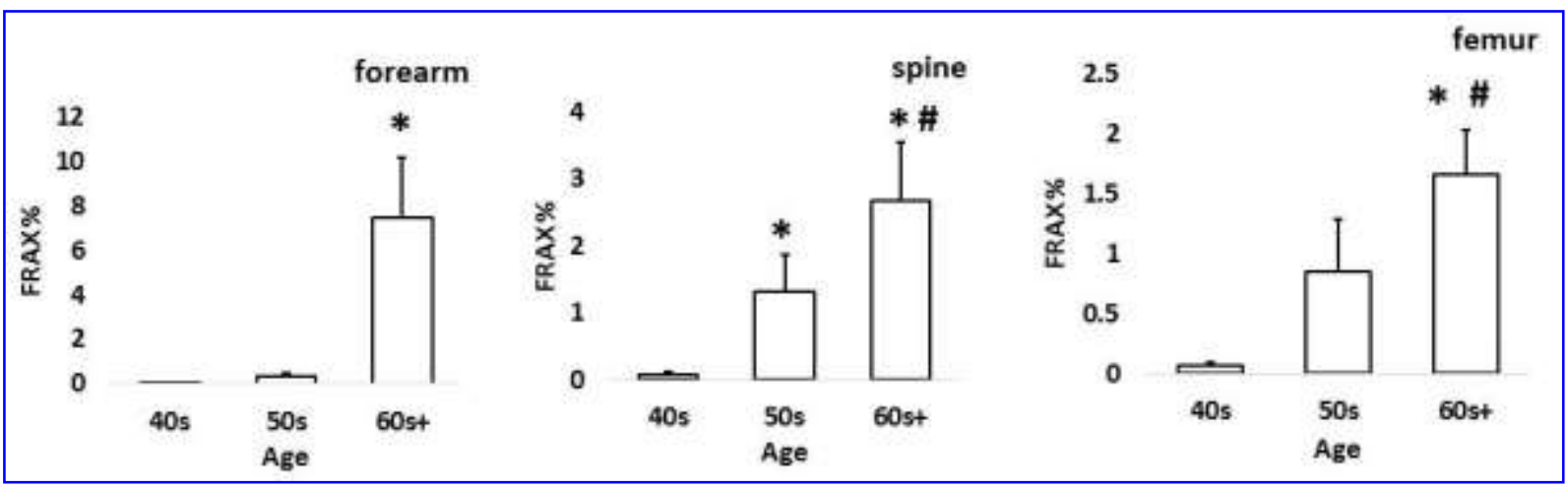

Fig. 2. Organ FRAX values as calculated from $T$-score of radius, spine and femur of hyperthyroid women of different ages. Data are presented as mean \pm SEM of $N=5$ from each age category. * denotes significantly different value from the value of age 40s, and \# from the value of age 50s.

The FRAX tool output includes both hip and major fracture ten year probability of fracture (\%). The Major FRAX results in the fracture probability for spine, femur and shoulder, although it uses the femur data only. Here, what will be the major FRAX, if it is calculated from data of the other organs? In case of hypothyroidism, figure 3 shows that major FRAX results were more or less similar, when calculated from data of forearm, spine or femur, and also similar to the FRAX calculated from summated data of all measured segments together (total). However, this summated result was more close to that from the femur. 


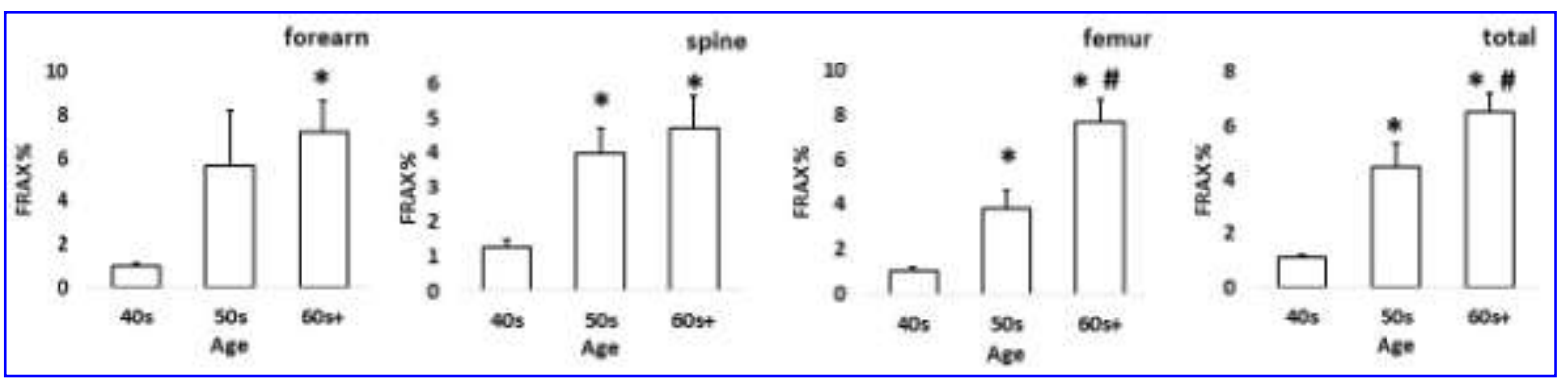

Fig. 3. Major osteoporotic FRAX values as calculated from T-score of different segments from hypothyroid women of different ages. Data are presented as mean \pm SEM of $\mathrm{N}=5$ from each age category. * denotes significantly different value from the value of age $40 \mathrm{~s}$, and \# from the value of age $50 \mathrm{~s}$.

Similar results were obtained for major osteoporotic FRAX of hyperthyroid female patients. FRAX value of each age category was significantly higher than that of the younger category. Similar results were obtained for FRAX values calculated from different organs. FRAX calculated from the collective data of all organs (total, Fig. 4) was also similar to that calculated from each single organ.

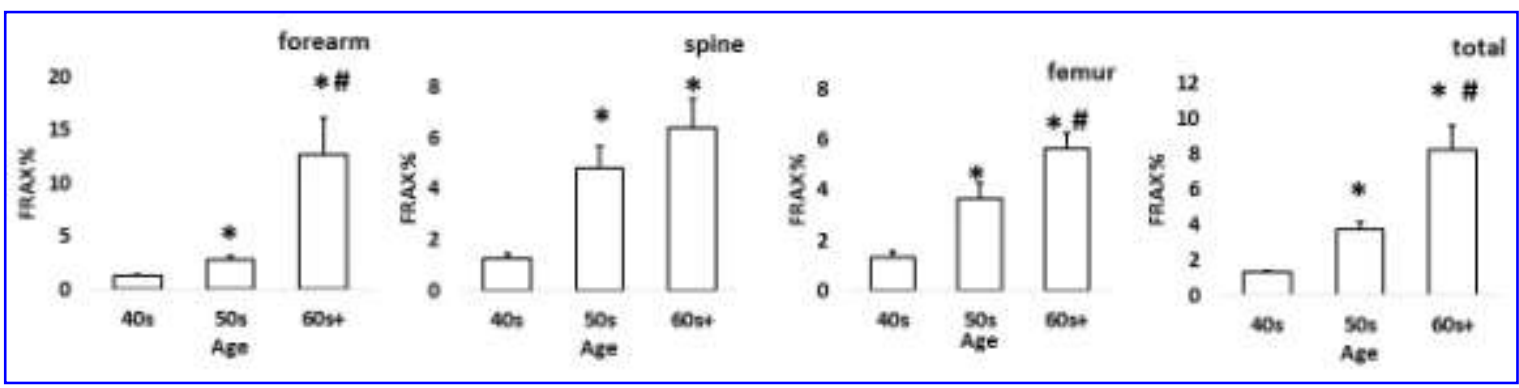

Fig. 4. Major osteoporotic FRAX values as calculated from T-score of different segments from hyperthyroid women of different ages. Data are presented as mean \pm SEM of $\mathrm{N}=5$ from each age category. * denotes significantly different value from the value of age $40 \mathrm{~s}$, and \# from the value of age $50 \mathrm{~s}$.

When FRAX values of different organs have been compared for the same age category (Table 1), no significant differences have been observed among them (ANOVA $\mathrm{p}>0.05$ ). This was valid for both hypo- and hyperthyroidism. Taken together, these results may indicate that BMD or T-score data from different organs can be recruited to calculate FRAX value of the corresponding organ.

Table 1. Comparison of FRAX values of different measured segments in hypothyroid and hyperthyroid women of different ages.

\begin{tabular}{lllll}
\hline Group & Age & Forearm & Spine & Femur \\
\hline \multirow{3}{*}{ hypothyroid } & $40 \mathrm{~s}$ & $0.06 \pm 0.03$ & $0.13 \pm 0.03$ & $0.1 \pm 0.04$ \\
& $50 \mathrm{~s}$ & $2.64 \pm 1.91$ & $1.00 \pm 0.38$ & $1.01 \pm 0.43$ \\
& $60 \mathrm{~s}+$ & $1.94 \pm 0.94$ & $1.24 \pm 0.63$ & $2.42 \pm 0.71$ \\
\hline \multirow{3}{*}{ hyperthyroid } & $40 \mathrm{~s}$ & $0.07 \pm 0.03$ & $0.08 \pm 0.04$ & $0.06 \pm 0.03$ \\
& $50 \mathrm{~s}$ & $0.32 \pm 0.13$ & $1.32 \pm 0.54$ & $0.84 \pm 0.45$ \\
& $60 \mathrm{~s}+$ & $4.27 \pm 1.66$ & $2.68 \pm 0.87$ & $1.66 \pm 0.36$ \\
\hline
\end{tabular}

Data are presented as mean \pm SEM. Statistical analysis by ANOVA: $p>0.05$ for all cases. 
Noreya M. Abeed et al.

\section{DISCUSSION}

The FRAX is increasingly being used as a guide for clinical decisionmaking, and FRAX models are currently available for 63 countries and in 32 languages, covering $79 \%$ of the world population aged 50 years or older (WHO, 2015). As fracture probability differs markedly within and across regions of the world (Radominski et al., 2017), the existing FRAX models were calibrated to the epidemiology of fracture and death in individual nations.

Since the release of the FRAX, there have been questions related to the limitation of the epidemiological studies that served as database for its calibration, as well as questions regarding whether or not those facts could diminish the relevance of the FRAX tool in the clinical practice. Information about the epidemiology of fractures in Egypt is not available. The FRAX tool requires only BMD for its input to result mainly in hip fracture risk assessment as output. Meanwhile, DXA scanning offer the data of spine and forearm BMD, which can be used to assess fracture specifically in spine and shoulder. The management strategy may require the consideration of these two additional data, instead of calculations depending on femur BMD only.

In the present study, fracture risk in pre- and postmenopausal women was examined among women aged 40 years or older who had hypo- and hyperthyroidism. Generally, the results revealed that FRAXscore was gradually increased by the age in hypo- and hyperthyroid women, although, the mean fracture risk was higher in hyperthyroid women in each of lumber spine and forearm, but the hip has the highest value in hypothyroidism than in hyperthyroidism, especially in the age group above 50 years.AllFRAX values were less than $10 \%$, so the fracture risk was low in thyroid dysfunction.

Numerous studies have investigated the consequences of altered thyroid function on fracture risk in adults (Heemstra et al., 2006; Taylor et al., 2013; Fröhlich and Wahl, 2015). The present results agree with the results by Vestergaard et al. (2009) who noted a temporary increase in fracture risk within the first 2 years after diagnosis of hypothyroidism. This fracture risk was mainly increased in the age group above 50 years. In another study of the same author was noticed that there was an increase in the risk of any fracture within the first 10 years after a diagnosis of hypothyroidism. Also, postmenopausal patients with subclinical hypothyroidism had higher FRAX scores and thus greater risk for low-trauma hip fracture than euthyroid postmenopausal women (Polovina et al., 2013).

The relationship between age and risk of hip fractures was reported by Vestergaard and Mosekilde (2003) who concluded that the risk of hip fractures was correlated positively with age at time of diagnosis of hyperthyroidism. Also, population studies have demonstrated an increased risk of fracture in thyrotoxicosis especially in post-menopausal women (Bauer et al., 2001; Vestergaard et al., 2005).The present results were parallel to the study in Pre- and postmenopausal women with subclinical hyperthyroidism which has higher FRAX scores and thus greater risk for low-trauma hip fracture than euthyroid premenopausal women (Polovina et al., 2015).

The results of the present study revealed similarities for FRAX calculation from femur data only or from corresponding organs. Thus, T-score data from different organs can be recruited to calculate FRAX value of the corresponding organ

In summary, the FRAX utilizes several known clinical risk factors in additionto BMD to calculate a patient's 10year fracture probability. The tool is calibrated by the best available epidemiological data, and can be 
continually upgraded as new data emerge, ensuring greater efficiency and ease-of-use in the clinical practice. Utilization of all available DXA results may help in this context.

\section{REFERENCES}

Albergaria, B.H.; Paula, F.J.A. (2019). The Algorhytm: FRAX Brazil. Rev Bras Ginecol Obstet. 41(8):467-468.

Bauer, D.C.; Hettinger, B.; Nevitt, M.C. and Stone, K.L. (2001). Study of Osteoporotic Fractures Research Group. Risk for fracture in women with low serum levels of thyroidstimulating hormone. Annals of Internal Medicine, 134 561-568.

Cummings, S.R.; Bates, D. and Black, D.M. (2002). Clinical use of bone densitometry.

J.A.M.A., 288(22):1889-1897.

Fröhlich, E. and Wahl, R. (2015). Mechanisms in endocrinology: impact of isolated TSH levels in and out of normal range on different tissues. Eur J Endocrinol, 174:R29-R41.

Heemstra, K.A.; Hamdy, N.A.; Romijn, J.A. and Smit, J.W. (2006). The effects of thyrotropin suppressive therapy on bone metabolism in patients with well-differentiated thyroid carcinoma. Thyroid, 16:583-591.

Kanis, J.A.; Borgström, F. and De Laet, C. (2005): Assessment of fracture risk. Osteoporos Int., 16 (6): 581589.

Kanis, J.A.; McCloskey, E.V.; Johansson, H.; Oden, A.; Ström, O. and Borgström, F. (2010). Development and use of FRAX in osteoporosis. Osteoporos Int., 21 (Suppl. 02): S407-S413.

WHO, World Health Organization Scientific Group (2007). Assessment of Osteoporosis at the Primary Health-Care Level. Summary Report of a WHO Scientific Group. Geneva: WHO;
Sheffield: University of Sheffield; https://www.sheffield.ac.uk/FRAX/ pdfs/WHO_Technical_Report.pdf.

Polovina, S.; Micić, D.; Miljić, D.; Milić, N.; Micić, D. and Popović, V. (2015). The Fracture Risk Assessment Tool (FRAX score) in subclinical

hyperthyroidism.Vojnosanit Pregl.,72(6):510-6

Polovina, S.; Popovic, V.; Duntas, L.; Milic, N. and Micic, D. (2013). Frax score calculations in postmenopausal women with subclinical hypothyroidism. Hormones (Athens), 12(3):439-48.

Radominski, S.C.; Bernardo, W.; Paula, A.P. , et al. (2017). Brazilian guidelines for the diagnosis and treatment of postmenopausal osteoporosis. Rev Bras Reumatol Engl Ed., 57 (Suppl. 02) 452-466

Tanner, S.B. and Moore, C.F.Jr. (2012). A review of the use of dual-energy $\mathrm{X}$ ray absorptiometry (DXA) in rheumatology. Rheumatol, 11, 4:99-107.

Taylor, P.N.; Razvi, S.; Pearce, S.H. and Dayan, C.M. (2013). Clinical review: a review of the clinical consequences of variation in thyroid function within the reference range. J. Clin. Endocrinol. Metab., 98: 35623571.

Vestergaard, P. and Mosekilde, L. (2003). Hyperthyroidism, bone mineral, and fracture risk-a meta-analysis. Thyroid, 13:585-593.

Vestergaard, P.; Weeke, J.; Hoeck, H.; Nielsen, H.; Rungby, J.; Rejnmark, L.; Laurberg, P. and Mosekilde, L. (2000). Fractures in patients with primary idiopathic hypothyroidism. Thyroid, 10:335-340.

Vestergaard, P.; Rejnmark, L. and Mosekilde, L. (2005). Influence of hyper- and hypothyroidism, and the effects of treatment with antithyroid drugs and 
Noreya M. Abeed et al.

levothyroxine on fracture risk. Calcif. Tissue Int., 77(3): 139-144.

Zerbini, C.A.; Szejnfeld, V.L.; Abergaria,

B.H.; McCloskey, E.V.; Johansson,

H. and Kanis, J.A.
(2015).Incidence of hip fracture in Brazil and the development of a FRAX model. Arch Osteoporos, 10: 224.

$$
\text { تقييم مخاطر كسور العظام فى مريضات الغدة الارقية في دمياط }
$$

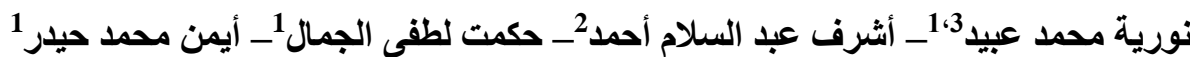
1 - كلية العلوم، جامعة دمياط، 2 - كلية الطب جامعة الأز هر بدمياط ، 3 - كلية التقنية الطبية، جامعة طر ابلس، ليبيا

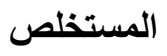

تكمن الأهمية الاكلينيكية لهثاشة العظام فى زيادة معدل الكسور في العظام. تعد أداة تقييم مخاطر الكسر هي الأداة المستخدمة على نطاق واسع لحساب مخاطر الكسور. تستخدم أداة

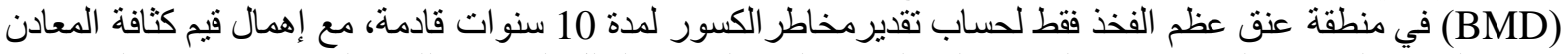

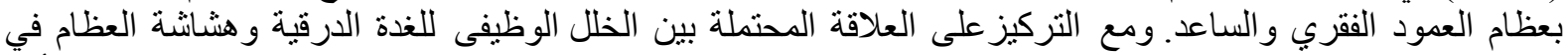

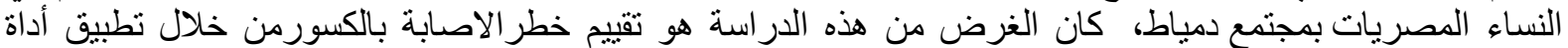

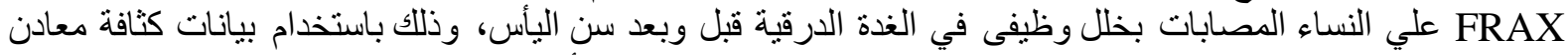

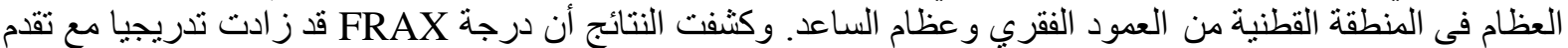

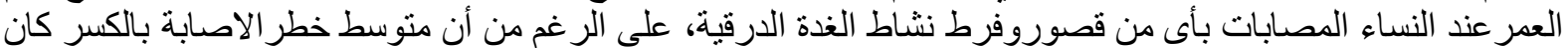

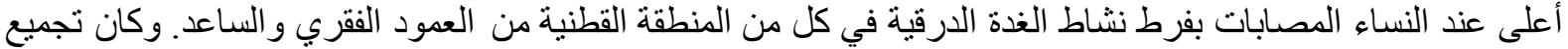

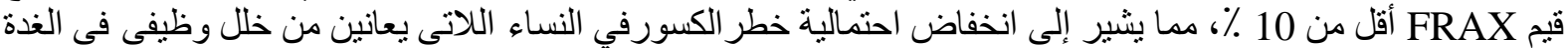

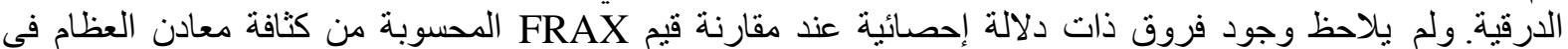

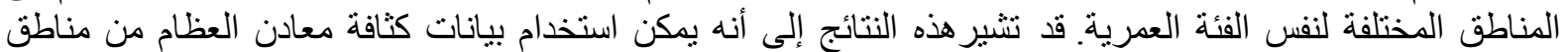

